\title{
Long-term Efficacy of Combined Treatment in Patients with Idiopathic Achalasia
}

\author{
Maryam Jameshorani ${ }^{1,2}$, Amir Anushiravani ${ }^{1}$, Narges Fazlollahi ${ }^{1}$, Ahmad Hormati ${ }^{3}$, Mohammad Amani ${ }^{1}$, \\ Javad Mikaeli ${ }^{3, *}$
}

1. Digestive Disease Research Institute, Shariati Hospital, Tehran University of Medical Sciences, Tehran, Iran

2. Department of Internal Medicine, School of Medicine Metabolic Diseases Research Center, Valiasr-e-Asr Hospital, Zanjan University of Medical Sciences, Zanjan, Iran

3. Gastroenterology and Hepatology Disease Research Center, Qom University of Medical Sciences, Shahid Beheshti Hospital, Qom, Iran

\section{* Corresponding Author:}

Javad Mikaeli, MD

Digestive Diseases Research Institute, Tehran University of Medical Sciences, Shariati Hospital: Karegar Shomali Ave, Tehran, Iran, postal code: 1411713135

Tel: + 982182415000

Fax: + 982182415400

Email:mikaeli@ams.ac.ir

Received: 20 Jun. 2020

Accepted: 03 Nov. 2020

\section{ABSTRACT}

\section{BACKGROUND}

Several treatment strategies are available to treat achalasia. Although combined therapy has been used for several years, there are limited data on long-term outcomes. We aimed to determine its long-term efficacy in patients who were resistant or those with rapid relapse.

\section{METHODS}

In this prospective study, we reviewed the records of 1100 patients with achalasia, who were candidates for pneumatic balloon dilatation (PBD) in our center from 1996 to 2018. We enrolled 197 patients resistant to initial treatment or with rapid relapse of symptoms after three sessions of PBD. Clinical evaluation and time barium esophagogram (TBE) were done before treatment, a month afterward, and when clinical symptoms increased in order to confirm relapse, and at the end of follow-up.

\section{RESULTS}

A total of 168 patients accepted combined therapy. The mean duration of follow-up was 9.04 years. Achalasia symptom score (ASS) dropped from 10.82 to 3.62 a month after treatment and was 3.09 at the end of the follow-up ( $p=0.0001$ and 0.001). TBE had a decrease in mean height of barium one month after treatment ( 9.23 vs. 5.10, $p=$ $0.001)$, and this reduction persisted until the end of follow-up $(3.39, p=0.001)$. Vantrappen score at the end of the follow-up showed 56 patients in excellent, 51 in good, 33 in moderate, and 14 in poor condition ( $89 \%$ acceptable response rate).

\section{CONCLUSION}

Our results showed the long-term efficacy of combined treatment in patients with achalasia who otherwise had to undergo a high-risk and costly procedure, which makes it a safe and effective alternative for myotomy.

\section{KEYWORDS :}

Therapeutics, Esophageal achalasia, Long-term care

Please cite this paper as:

Jameshorani M, Anushiravani A, Fazlollahi N, Hormati A, Amani M, Mikaeli J. Long-term Efficacy of Combined Treatment in Patients with Idiopathic Achalasia. Middle East J Dig Dis 2021;13:21-26. doi: 10.34172/mejdd.2021.199.

\section{INTRODUCTION}

Idiopathic achalasia is a primary chronic motor disorder of the esophagus with 


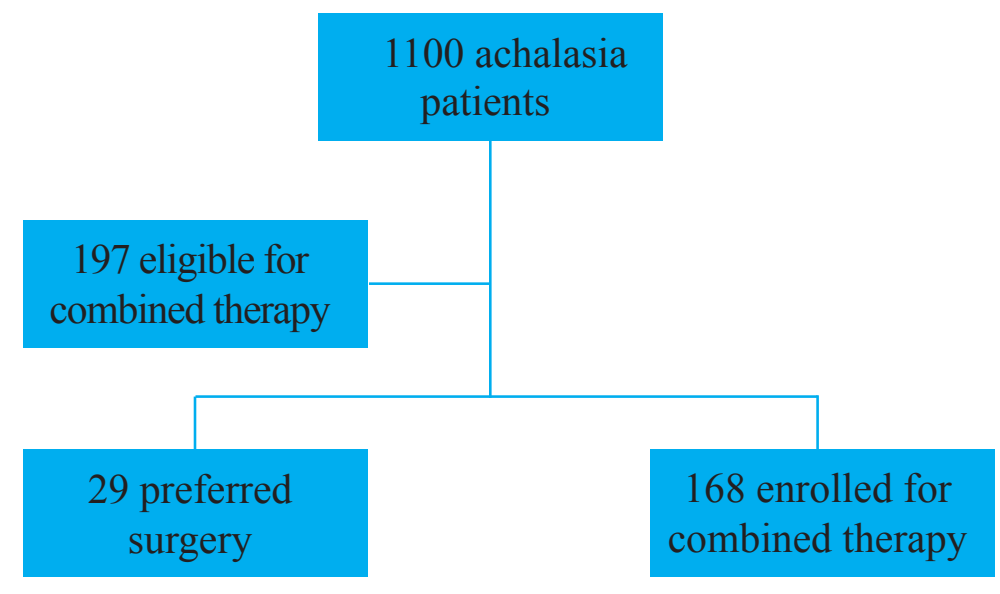

Fig.1: Patients arrangement

incomplete or absent relaxation of the lower esophageal sphincter (LES) during swallowing and aperistalsis of the esophageal body. ${ }^{1}$ Histopathological data of the achalasic esophagus showed a loss of ganglionic cells in the myenteric (Auerbach's) plexus. The primary pathophysiology seems to be losing inhibitory ganglion cells, which secrete nitric oxide (NO) and vasoactive intestinal peptide (VIP), and the persistence of cholinergic stimulatory cells. ${ }^{2}$

Several treatment strategies are available to decrease the LES pressure, such as pharmacological drugs, pneumatic balloon dilatation (PBD), botulinum toxin (BT), ethanolamine oleate injections, and endoscopic or surgical myotomy. ${ }^{3,4}$ PBD is a common method of treatment that provides good to excellent symptomatic relief with a response rate of approximately $90 \%$. Several studies have compared the efficacy of PBD, BT, and the combined method (PBD 1 month after BT injection). PBD is effective in most of the patients with achalasia; however, the combined method (BT and PBD) provides a better response rate. ${ }^{5} \mathrm{BT}$ induces LES relaxation by inhibiting acetylcholine release from the nerve endings and decreasing unopposed cholinergic stimulation of the LES. Studies have shown a low sustained effect, with only about $30 \%$ response at 12 months. ${ }^{6}$ Heller myotomy is a safe and effective second-line treatment for achalasia (after PBD or BT injections), but it seems to be less satisfactory in those previously treated with BT in longterm evaluations. ${ }^{7}$

Although combined therapy has been used for several years as achalasia treatment, there are limited data on the long-term outcomes of patients treated with this procedure. Hence, we aimed to determine the long-term efficacy of BT injection before PBD in controlling symptoms of achalasia in patients who were resistant to therapy or those with rapid relapse.

\section{MATERIALS AND METHODS}

\section{Patients}

In this prospective study, we reviewed the records of 1100 patients previously diagnosed with achalasia who were candidates for PBD in our center from January 1996 to May 2018. The diagnosis was established based on clinical, radiological, endoscopic, as well as manometric criteria. Exclusion criteria were age $<18$ years, cardiovascular disability (functional class III or IV), and coagulopathy.

Informed consent was obtained from all patients after a full discussion of the risks, benefits, and alternatives.

\section{Study design}

Patients resistant to initial treatment and those with rapid relapse of symptoms after three sessions of PBD were enrolled. Achalasia Symptom Score (ASS) $>4$ and retention of barium in timed barium esophagogram (TBE) during six months after the last PBD were used to define resistance or rapid relapse.

Overall, 197 patients with achalasia were enrolled in our study (Figure1). Surgical myotomy and combined therapy were completely explained to each patient. Of them, 168 patients preferred combined therapy (PBD 1 month after BT) with the following protocol:

All patients had BT injection (Dysport 500 units) into 
Table 1: Cardinal symptoms score in achalasia

\begin{tabular}{lcccc}
\hline Symptom & Each meal & Daily & Weekly & None \\
\hline $\begin{array}{l}\text { Dysphagia to } \\
\text { solids }\end{array}$ & 3 & 2 & 1 & 0 \\
\hline $\begin{array}{l}\text { Dysphagia to } \\
\text { liquids }\end{array}$ & 3 & 2 & 1 & 0 \\
\hline $\begin{array}{l}\text { Active } \\
\text { regurgitation }\end{array}$ & 3 & 2 & 1 & 0 \\
\hline Symptom & Daily & Weekly & Monthly & None \\
\hline $\begin{array}{l}\text { Passive } \\
\text { regurgitation }\end{array}$ & 3 & 2 & 1 & 0 \\
\hline Chest pain & 3 & 2 & 1 & 0 \\
\hline
\end{tabular}

the LES, and PBD was performed a month later using a 35-mm Rigiflex balloon. Clinical assessment and TBE were performed one month after the combined therapy, and patients were followed up by clinical evaluation every 6 months. If the ASS increased to more than four, TBE was repeated to confirm the relapse. Treatment was repeated for those with barium retention at 5 minutes.

Patients developing clinical relapse in less than a year underwent retreatment with BT and PBD with a 40-mm Rigiflex balloon. Those with relapse after a year received retreatment with BT and PBD, again using a 35-mm Rigiflex balloon. The study protocol was reviewed and approved by the Ethics Committee of Digestive Disease Research Center at Tehran University of Medical Sciences, and informed consent was obtained from all patients.

\section{Clinical evaluation}

Clinical evaluation was done prior to treatment and a month afterward, being repeated every 6 months. The severity of symptoms was evaluated based on five clinical symptoms: solid and liquid dysphagia, active and passive regurgitation, and chest pain. The severity of each of these symptoms was scored on a scale of 0-3 (Tables 1,2). ${ }^{9}, 10$ The highest obtainable score was 18 , where the clinical response was defined as a symptom score $\leq 4$ without severe dysphagia (accompanied by regurgitation), and those with a symptom score $>4$ were considered to have relapsed.

Patients' symptoms were evaluated by Vantrappen score at the end of their follow-up, expressed as: excellent, good, moderate, or poor (Table 3).

\section{Timed barium esophagogram}

TBE was performed before combined therapy, one month after $\mathrm{PBD}$, and anytime during follow-up when clinical symptoms increased (ASS $>4$ ) in order to confirm relapse and at the end of follow-up. Patients were told to swallow $200 \mathrm{~mL}$ of low-density barium sulfate suspension ( $81 \%$ weight/volume) while standing for over 30-45 seconds. Radiographs from the left posterior oblique view were taken at 1,3 , and 5 minutes afterward. Barium column height, defined as the distance from the most distal part of the esophagus to the proximal barium level, was measured in centimeters. The volume of retained barium $(\mathrm{mL})$ was calculated as follows:

(mean radius) $2 \times 3.14 \times$ height of column.

The initial TBE in our patients showed barium retention at 1, 3, and 5 minutes, a dilated esophagus, and a beak-like narrowing at the gastroesophageal (GE) junction. Differences in retained barium height and volume at 5 minutes before and after treatment were calculated.

\section{Botulinum toxin injection}

BT was injected one month before PBD. The LES was identified by visualizing the sphincter rosette at the squamocolumnar junction during esophagogastroduodenoscopy. 500 units of Clostridium botulinum type A toxin-hemagglutinin complex (Dysport; IPSEN, Berkshire, UK) diluted in $5 \mathrm{~mL}$ normal saline was injected through a 5 -mm sclerotherapy needle into the LES in ten injections of $0.5 \mathrm{~mL}$. These injections were done circumferentially at the GE junction up to 2 $\mathrm{cm}$ above the Z-line.

\section{Pneumatic balloon dilatation}

After a clear liquid diet for $48 \mathrm{~h}$ and an overnight fast, PBDs were performed using a 35-mm Rigiflex balloon. Following a complete esophagogastroduodenoscopy, balloon dilators were passed over a guidewire and were positioned in the middle of the LES using a video endoscopic guide. The balloons were gradually inflated up to $15 \mathrm{psi}$ in $30 \mathrm{~s}$, and this pressure was maintained for $60 \mathrm{~s}$. Afterward, esophagoscopy was repeated to assess the LES opening (relaxation) and any evidence of bleeding or perforation. They were discharged after a 6-h observation.

\section{Statistical analysis}


Table 2: Severity score of dysphagia for every swallow

\begin{tabular}{lcc}
\hline Severity & Score & Description \\
\hline No dysphagia & 0 & Normal passage of food from LES zone \\
\hline Mild dysphagia & 1 & Sensation or short delay of the passage of food from LES, without the need of water \\
\hline Moderate dysphagia & 2 & Need for water for passage of food from LES zone \\
\hline Severe dysphagia & 3 & Accompanied by passive or active regurgitation \\
\hline
\end{tabular}

Table 3: Vantrappen scoring system in achalasia

\begin{tabular}{lc}
\hline Class & Dysphagia \\
\hline Excellent & Completely free of symptoms \\
\hline Good & Dysphagia more than once a week lasting less than 2-3 min and not accompanied by regurgi-tation or weight loss \\
\hline Moderate & Dysphagia more than once a week or lasting 2-3 min or longer or accompanied by regurgita-tion or weight loss \\
\hline Poor &
\end{tabular}

Table 4: Achalasia Symptom Score before and after the combined therapy

\begin{tabular}{|c|c|c|c|}
\hline ASS & & Mean ( \pm SD) & $p$ value \\
\hline Before therapy & & $10.82 \pm 2.97$ & - \\
\hline 1 month & \multirow{3}{*}{ 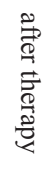 } & $3.62 \pm 3.57$ & 0.0001 \\
\hline 6 months & & $3.74 \pm 3.45$ & 0.0001 \\
\hline 12 months & & $3.09 \pm 3.15$ & 0.0001 \\
\hline
\end{tabular}

Descriptive statistics are provided as the median and interquartile range for age, symptom score, LES pressure, and barium height and volume. Quantitative variables are presented as medians and ranges. SPSS software version 21 was used for the statistical analysis. The Kaplan-Meier method was used to assess symptomatic recovery. $p$ values $<0.05$ were considered significant.

\section{RESULTS}

A total of 168 patients (90 men and 78 women; median age, 40 years), with previously diagnosed achalasia treated with combined therapy (PBD following injections of BT), were enrolled from January 1996 to May 2018. The mean duration of follow-up was 9.04 years. 14 patients had surgery during follow-up due to symptom relapse.

Up to 2008, manometry was done using conventional manometry, and we had 85 patients with the classic and 6 with the vigorous type. After implementing high-resolution manometry in our center, the frequencies of achalasia subtypes were 13,62 , and 2 for types 1, 2, and 3, respectively. The mean LES resting pressure was $32.61 \mathrm{mmHg}$.

ASS dropped from 10.82 to 3.62 a month after treatment and was 3.09 at the end of follow-up ( $p=0.0001$ and 0.001$)$ (Table 4).

Timed barium esophagram had a decrease in the mean height of barium one month after treatment (9.23 vs. 5.10, $p=0.001)$, and this reduction persisted until the end of the follow-up (3.39, $p=0.001$, Table 5).

Vantrappen score was used to evaluate patients at the end of their follow-up, where 56 patients were in excellent, 51 in good, 33 in moderate, and 14 in poor condition (Table 6).

During follow-up, 109 patients underwent BT-PBD once $(64.8 \%), 49$ patients twice $(29.1 \%)$, and 10 patients three times $(5.9 \%)$. The rate of relapse was $35 \%$ during this long-term follow-up ( $p=0.042$ ) (Figure 2).

Interesting to note, among those who had surgery during follow-up (14 patients), five developed symptom relapse. BT-PBD was done again for them with excellent and good responses, according to the Vantrappen score, seen in three and two patients, respectively.

\section{DISCUSSION}

In our study, we showed that patients with idiopathic achalasia who were potential candidates for surgery were effectively treated in the long term with combined therapy, reducing the need for surgery in about $80 \%$ of cases.

Vantrappen scoring system was used to evaluate the response rate at the end of follow-up. About two-thirds of our patients showed excellent or good response to this treatment strategy (69\%). Since patients in the moderate class do not have severe dysphagia accompanied by regurgitation, we can consider them as partial responders, 
Table 5: Timed barium esophagram in combined treatment for patients with resistant and rapid relapse achalasia

\begin{tabular}{|c|c|c|c|}
\hline TBE Volume and Height & & Mean $( \pm$ SD) & $p$ value \\
\hline \multirow{3}{*}{ Volume (mL) } & Before therapy & $66.98 \pm 34.24$ & - \\
\hline & 1 month after therapy & $34.24 \pm 58.25$ & 0.001 \\
\hline & End of follow up & $22.02 \pm 50.48$ & 0.0001 \\
\hline \multirow{3}{*}{ Height (cm) } & Before therapy & $9.23 \pm 5.755$ & - \\
\hline & 1 month after therapy & $5.10 \pm 6.262$ & 0.01 \\
\hline & End of follow-up & $3.88 \pm 5.459$ & 0.001 \\
\hline
\end{tabular}

Table 6: Timed barium esophagram in combined treatment for patients with resistant and rapid relapse achalasia

\begin{tabular}{lcc}
\hline Vantrappen score & Frequency & Percentage \\
\hline Excellent & 56 & 36.36 \\
\hline Good & 51 & 33.11 \\
\hline Moderate & 33 & 21.42 \\
\hline Poor & 14 & 9.09 \\
\hline Total & 154 & 100.0 \\
\hline
\end{tabular}

and therefore, their response would also be acceptable, meaning a $90 \%$ response using combined therapy.

The strength of our study was a relatively long duration with a mean follow-up of over 9 years.

Treatment options for achalasia include pharmacologic agents, $\mathrm{PBD}$, intrasphincteric $\mathrm{BT}$ injection, and surgical or endoscopic myotomy. Peroral endoscopic myotomy (POEM) is less invasive than laparoscopic Heller's myotomy (LHM), and in a recent randomized trial, it was proven to be non-inferior to LHM in controlling achalasia symptoms 2 years in follow-up. ${ }^{8}$

Several studies have compared the efficacy of these different therapeutic strategies in treatment-naïve patients, but we did not find any study on the long-term efficacy of a non-surgical therapeutic method for resistant or rapid relapse cases of idiopathic achalasia.

A study revealed that PBD is more effective than BT injections, ${ }^{9}$ and $\mathrm{PBD}$ is an appropriate long-term treatment. Another study showed PBD as being a good treatment for long-term relief, and BT with a relatively short-term response..$^{10}$ In that study, we showed that combined therapy decreased the ASS by $76 \%$, compared with a $53 \%$ decrease in those undergoing only PBD with a mean follow-up of about 2 years. ${ }^{10}$ In this study, we have shown the longterm efficacy of the combined therapy for those with rapid relapse or resistant achalasia.

Ethanolamine oleate (EO) was shown to be effective

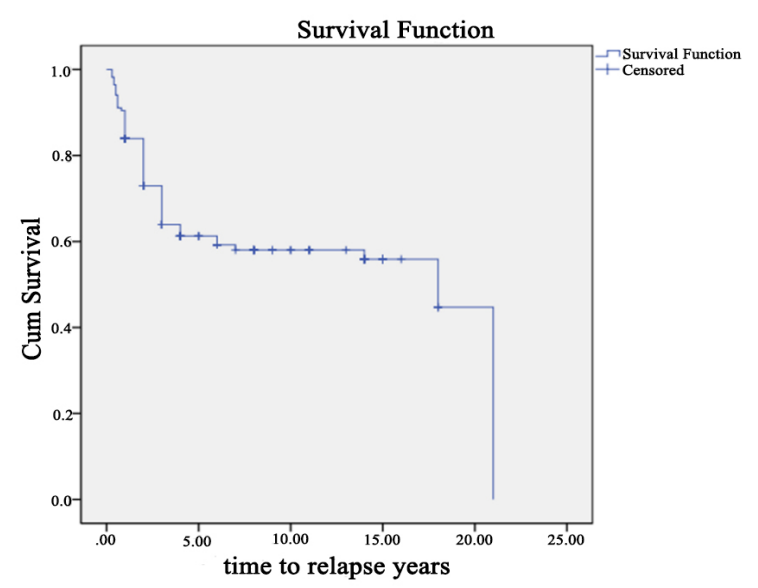

Fig.2: The rate of relapse after combined therapy in patients with achalasia

in resistant achalasia in 31 patients. It was injected in the LES every 2 weeks three times, and they were followed for a mean duration of 30 weeks. A good response was seen in 29 patients, in whom 12 had relapsed and were retreated with EO injections. Only minor adverse events occurred in this trial, and the authors concluded that EO injection could safely be used in the treatment of resistant idiopathic achalasia. ${ }^{11}$

A small group of patients (nine patients) with achalasia were treated with the combined therapy (250 units of Dysport followed 7 days later with PBD). They were followed every 3 months, and the longest symptom improvement was 3 years. Clinical and manometric improvement was seen in seven of their patients after one year. ${ }^{12}$

A randomized clinical trial conducted on 90 patients (in three equal groups) with achalasia revealed that those who received combined therapy (100 units of BT injected 15 days after $\mathrm{PBD}$ ) had a significantly better response rate at two years after treatment, compared with those who received only one of the therapeutic methods ( $56 \%$ compared with $35 \%$ and $13 \%$ for PBD and BT, respectively). ${ }^{13}$ We gave 
the combined therapy to 168 patients with rapid relapse and resistant achalasia, and they were followed up for over 9 years, with at least a partial response in about $90 \%$.

Kroupa and colleagues followed up treatment-naïve patients with achalasia for a mean duration of 48 months after using the combined therapy (200 units of BT with PBD 8 days later), and $72 \%$ had a satisfactory response at their last visit. They concluded that BT prior to PBD was effective in the long term with few complications such as heartburn, but this combined therapy was not superior to PBD alone. ${ }^{14}$ We enrolled 168 patients who were potential candidates for surgical myotomy. These patients were followed up for a mean duration of more than 9 years using the Vantrappen scoring system, ASS, and TBE. All three were significantly improved after the combined treatment with a relapse rate as low as $35 \%$. Even these patients had a good response after repeating the combined therapy. Only $18 \%$ showed a poor response to this treatment, with a poor Vantrappen score at the end of follow-up or having to do surgery.

To our knowledge, we have reported the longest followup of a combined treatment strategy in patients with achalasia and with resistance or rapid relapse after PBD. We enrolled a large population of these patients and followed them up with clinical symptoms and TBE. Our results determined the long-term efficacy of the combined treatment in patients with achalasia who otherwise had to undergo a high-risk and costly procedure, making it a safe and effective alternative for myotomy in the elderly and those with comorbidities.

\section{ETHICAL APPROVAL}

There is nothing to be declared.

\section{CONFLICT OF INTEREST}

The authors declare no conflict of interest related to this work.

\section{REFERENCES}

1. Mikaeli J, Islami F, Malekzadeh R. Achalasia: a review of Western and Iranian experiences. World J Gastroenterol 2009;15:5000-9. doi: 10.3748/wjg.15.5000.

2. Farrokhi F, Vaezi MF. Idiopathic (primary) achalasia. Orphanet J Rare Dis 2007;2:38. doi: 10.1186/17501172-2-38

3. Allescher HD, Storr M, Seige M, Gonzales-Donoso R,
Ott R, Born P, et al. Treatment of achalasia: botulinum toxin injection vs. pneumatic balloon dilation. A prospective study with long-term follow-Up. Endoscopy 2001;33:1007-17. doi: 10.1055/s-2001-18935.

4. Mikaeli J, Fazel A, Montazeri G, Yaghoobi M, Malekzadeh R. Randomized controlled trial comparing botulinum toxin injection to pneumatic dilatation for the treatment of achalasia. Aliment Pharmacol Ther 2001;15:138996. doi: 10.1046/j.1365-2036.2001.01065.x.

5. Bakhshipour A, Rabbani R, Shirani S, Soleimani HA, Mikaeli J. Comparison of pneumatic dilation with pneumatic dilation plus botulinum toxin for treatment of achalasia. Acta Med Iran 2010;48:107-10.

6. BassottiG,Annese V. Review article: pharmacological options in achalasia. Aliment Pharmacol Ther 1999;13:13916. doi: 10.1046/j.1365-2036.1999.00645.x.

7. Portale G, Costantini M, Rizzetto C, Guirroli E, Ceolin M, Salvador R, et al. Long-term outcome of laparoscopic Heller-Dor surgery for esophageal achalasia: possible detrimental role of previous endoscopic treatment. $J$ Gastrointest Surg 2005;9:1332-9. doi: 10.1016/j.gassur.2005.10.001

8. Werner YB, Hakanson B, Martinek J, Repici A, Rahden BHA, Bredenoord AJ, et al. Endoscopic or Surgical Myotomy in Patients with Idiopathic Achalasia. $N$ Engl $\mathrm{J}$ Med 2019;381:2219-29. doi:10.1056/NEJMoa1905380

9. Bansal R, Nostrant TT, Scheiman JM, Koshy S, Barnett JL, Elta GH, et al. Intrasphincteric botulinum toxin versus pneumatic balloon dilation for treatment of primary achalasia. J Clin Gastroenterol 2003;36:209-14. doi: 10.1097/00004836-200303000-00005.

10. Mikaeli J, Yaghoobi M, Montazeri G, Ansari R, Bishehsari F, Malekzadeh R. Efficacy of botulinum toxin injection before pneumatic dilatation in patients with idiopathic achalasia. Dis Esophagus 2004;17:2137. doi: $10.1111 / \mathrm{j} .1442-2050.2004 .00410 . x$.

11. Niknam R, Mikaeli J, Fazlollahi N, Mahmoudi L, Mehrabi N, Shirani S, et al. Ethanolamine oleate as a novel therapy is effective in resistant idiopathic achalasia. Dis Esophagus 2014;27:611-6. doi: 10.1111/dote.12122.

12. Hep A, Dolina J, Plottova Z, Valek V, Novotny I, Kala $Z$. [Is complex therapy of achalasia using botulinum toxin combined with balloon dilatation an effective approach?]. Bratisl Lek listy 2000;101:433-7.

13. Zhu Q, Liu J, Yang C. Clinical study on combined therapy of botulinum toxin injection and small balloon dilation in patients with esophageal achalasia. Dig Surg 2009;26:493-8. doi: 10.1159/000229784.

14. Kroupa R, Hep A, Dolina J, Valek V, Matyasova Z, Prokesova J, et al. Combined treatment of achalasia botulinum toxin injection followed by pneumatic dilatation: long-term results. Dis Esophagus 2010;23:100-5. doi: 10.1111/j.1442-2050.2009.01005.x. 